\title{
Biosafety Measures in the Laboratory during Handling of Specimens of Suspected COVID-19 Patients
}

\author{
Zakir Hossain Habib ${ }^{1}$, Ahmed Nawsher Alam²
}

\begin{abstract}
${ }^{1}$ Principal Scientific Officer, Institute of Epidemiology, Disease Control \& Research, Dhaka, Bangladesh; ${ }^{2}$ Principal Scientific Officer, Institute of Epidemiology, Disease Control \& Research, Dhaka, Bangladesh
\end{abstract}

[Received: 26 March 2020; Accepted: 2 April 2020; Published: 25 April 2020]

Appropriate biosafety practices are prime concern for the laboratories working with specimens of suspected COVID-19 patients. Clinicians should notify laboratory when specimens are submitted from a patient with suspected or confirmed COVID-19. This should be done through proper completion of request forms or electronic test requesting systems, or by direct communication with the clinical diagnostic laboratory. It is possible that clinicians may not have considered COVID-19 as a potential diagnosis prior to sending specimens to the laboratory. Good laboratory practice, including the use of standard biological safety precautions, regular training of staff, and the use of standard operating procedures, will help minimize potential risks. Risk assessment is very essential during laboratory works. Therefore, clinical laboratories must perform their own risk assessments for handling biological specimens from patients with suspected or confirmed COVID-19. Risk assessments and mitigation measures are dependent on: the procedures performed, Identification of the hazards involved in the process and/or procedures, the competency level of the personnel who perform the procedures, the laboratory equipment and facility and the resources available.

\section{Personal Protections}

Laboratory staff must wear personal protective equipment (PPE) when conducting work in the laboratory. PPE must be removed on leaving the laboratory and hygiene practices including hand washing must be rigorously maintained. PPE must include disposable gloves and a long sleeve laboratory coat preferably with elasticated or fitted cuffs. For procedures with a high likelihood to generate aerosols or droplets, use either a certified Class II Biological Safety Cabinet (BSC) or additional precautions to provide a barrier between the specimen and personnel. Examples of these additional precautions include surgical mask or face shield, or other physical barriers like a splash shield, centrifuge safety cups and sealed centrifuge rotors to reduce the risk of exposure to laboratory personnel. It should be borne in mind that masks or respirators are not an appropriate substitute for processing samples in a biological safety cabinet when there is a risk of aerosols being generated.

Non-propagative diagnostic laboratory work (for example, sequencing, nucleic acid amplification test [NAAT]) should be conducted at a facility using procedures equivalent to Biosafety Level 2 (BSL2). Propagative work (for example, virus culture, isolation or neutralization assays) should be conducted at a containment laboratory with inward directional airflow (BSL-3).

\section{Routine Laboratory Blood Tests}

Routine laboratory blood tests can be carried out in auto-analyzers using standard practices and procedures at Containment level-2 (CL-2), but only after a suitable and sufficient risk assessment has been conducted which considers the potential for the generation of infectious aerosols. Autoanalyzers should be disinfected following standard procedures after sample processing and before scheduled maintenance in accordance with manufacturers' recommendations. 
Some auto-analyzer protocols for routine laboratory tests may require specimen tubes to be opened first or initial processing of the sample to be performed. Evidence suggests that capping and uncapping of samples is not a high-risk aerosol generating procedure which is dependent on the cap and tube design. These factors must be considered in a suitable and sufficient risk assessment which also considers if the sample needs to be centrifuged, vortexes or pipetted manually. The risk assessment must include consideration of whether a BSC needs to be used.

\section{Use of Biosafety Cabinet}

Following completion of a suitable and sufficient risk assessment, the works with samples potentially containing SARS-CoV-2 may be conducted in a Biological safety cabinet at Containment Level 2 are preparation of specimens for molecular testing (for example respiratory virus PCR) prior to sample inactivation, division, aliquoting, or diluting of respiratory tract specimens, faecal specimens, urine specimens, and tissue specimens in which virus has not been inactivated, inoculation of bacterial or fungal culture media from high risk patients, urine antigen testing (such as for detection of Legionella pneumophila or Streptococcus pneumoniae), rapid antigen tests of respiratory tract specimens, processing of any non-inactivated specimen that might result in the generation of aerosols, preparation and fixing (chemical or heat) of smears for microscopy, haematological or immunological work, rapid diagnostic tests for malaria parasites.

Where risk assessment has identified that work should be conducted within an Biological safety cabinet at CL-2, still it implies that laboratory works should be performed by only fully trained and competent staff. In addition to this, training should be provided appropriate to the level of risk and the complexity of the procedures being undertaken, inactivation methods must be in place before removal of sample from BSC. These methods must be validated to ensure effectiveness of the method for example through use of a surrogate marker, effective emergency procedures, including methods for dealing with spillage, are in place and waste disposal routes for samples are appropriate for high risk group 3 samples.

\section{Centrifugation of Specimens}

Centrifugation of specimens with infectious potential must be performed using sealed centrifuge rotors or sample cups which are loaded and unloaded in a BSC. During movement of samples within the laboratory, external surfaces of specimen containers and vials must be decontaminated using a disinfectant with proven activity against enveloped viruses, prior to their removal from the BSC. Appropriate care should be taken to avoid accidental contamination of the exterior surfaces of all vessels and containers, regardless of containment level.

\section{Packaging \& Transport}

During packaging and transport of samples, final packaging of potentially infectious specimens (for example, to send to a reference laboratory) may be performed at Containment Level 2 if the specimens are already contained within a sealed and decontaminated primary container. Cultured samples for research or calibration must be transported in accordance with Category A transportation regulations. All potentially infectious samples must be transported in accordance with Category B transportation regulations (UN3373). Transport using same day delivery courier is recommended for all sample types, except for cultured samples for research or calibration which must be transported by an approved Category A carrier.

\section{Clinical Waste Management}

During cleaning and handling of waste, irrespective of the containment level, work surfaces and equipment must be decontaminated after specimens have been processed. Attention should be paid to all surfaces that may have come into contact with specimens or specimen containers. Disinfectant solution or disinfectant wipe should be used with proven activity against enveloped viruses, in accordance with local policies and following the manufacturer's instructions.

The clinical waste of suspected Covid-19 patients should be disposed of according to local and national policies appropriate to the categorization of the waste. Waste from auto-analyzers is unlikely to pose a significant risk due to the low sample volume and dilution steps, therefore special waste disposal precautions are not recommended for autoanalyzer waste.

\section{Emergency procedures}

Emergency procedures must be reviewed according to the results of risk assessments. Fumigation of laboratory spaces will not be possible where 
samples are spilt outside of Containment Level 3, therefore consideration must be given to what to do in the event of a spillage, and how effective decontamination of the area will be completed. Staff must be trained on updated emergency procedures.

\section{Conclusion}

In the context of maintaining service delivery, it is recommended that urgent and essential clinical diagnostic tests are not postponed pending the results of SARS-CoV-2 (COVID-19) testing, provided this is consistent with the risk assessment for the planned work and that standard containment measures are in place.
[Bangladesh Journal of Infectious Diseases, April 2020;7(suppl_1):S66-S68]

Correspondence: Dr. Zakir Hossain Habib, Principal Scientific Officer (PSO), Institute of Epidemiology, Disease Control \& Research (IEDCR), Mohakhali, Dhaka 1212, Bangladesh: Email: parashhabib@gmail.com; Cell No.: $+8801711109160$

Conflict of Interest: Authors have no competing interests.

How to cite this article: Habib $\mathrm{ZH}$, Alam AN. Biosafety Measures in the Laboratory during Handling of Specimens of Suspected COVID-19 Patients. Bangladesh J Infect Dis 2020;7(suppl_1):S63-S65

Copyright: ( $) 2020$. Habib and Alam. Published by Bangladesh Journal of Infectious Diseases. This article is published under the Creative Commons CC BY-NC License (https://creativecommons.org/licenses/by-nc/4.0/). This license permits use, distribution and reproduction in any medium, provided the original work is properly cited, and is not used for commercial purposes 